\title{
Designing a Digital Archive for Indigenous People
}

\author{
Understanding the Double Sensitivity of Design
}

\author{
Fatemeh Moradi* \\ Department of Informatics, Umeå university, Umeå, \\ Sweden \\ fatemeh.moradi@umu.se \\ Hanna Nordin \\ Department of Informatics, Umeå university, Umeå, \\ Sweden, hanna.nordin@umu.se se
}

\begin{abstract}
In this paper we present our work on the design and evaluation of a web-based digital archive. The aim of this research project was to explore ways of enabling easy access to materials about their cultural heritage for indigenous people. In this project we worked in close collaboration with the Sami people across brainstorming sessions, design workshops, prototype development, and user tests. During this process we became aware of two intertwined sensitivities, i.e. a cultural sensitivity and a design sensitivity - and we refer to this as a "double sensitivity". The data recorded from the interviews and the participants' interaction with the prototype were analyzed using thematic analysis as the methodological approach. Our results pointed at five main code clusters including: tonality of the design, usability, sociability, ethical considerations and technical errors. In this paper we discuss these findings, and we suggest that our results, and the proposed notion of "double sensitivity" contributes important research on human computer interaction (HCI) design for indigenous people.
\end{abstract}

\section{CCS CONCEPTS}

- Human-centered computing; • Human computer interaction (HCI); • Empirical studies in HCI;

\section{KEYWORDS}

Cultural sensitivity, design sensitivity, digital archive, indigenous people

\section{ACM Reference Format:}

Fatemeh Moradi*, Linnea Ỏhlund, Hanna Nordin, and Mikael Wiberg. 2020. Designing a Digital Archive for Indigenous People: Understanding the Double Sensitivity of Design. In Proceedings of the 11th Nordic Conference on Human-Computer Interaction: Shaping Experiences, Shaping Society (NordiCHI '20), October 25-29, 2020, Tallinn, Estonia. ACM, New York, NY, USA, 11 pages. https://doi.org/10.1145/3419249.3420174

Permission to make digital or hard copies of all or part of this work for personal or classroom use is granted without fee provided that copies are not made or distributed for profit or commercial advantage and that copies bear this notice and the full citation on the first page. Copyrights for components of this work owned by others than ACM must be honored. Abstracting with credit is permitted. To copy otherwise, or republish, to post on servers or to redistribute to lists, requires prior specific permission and/or a fee. Request permissions from permissions@acm.org.

NordiCHI '20, October 25-29, 2020, Tallinn, Estonia

(c) 2020 Association for Computing Machinery.

ACM ISBN 978-1-4503-7579-5/20/10 . \$15.00

https://doi.org/10.1145/3419249.3420174

\author{
Linnea Öhlund \\ Department of Informatics, Umeå university, Umeå, \\ Sweden \\ linnea.ohlund@umu.se \\ Mikael Wiberg \\ Department of Informatics, Umeå university, Umeå, \\ Sweden \\ mikael.wiberg@umu.se
}

\section{INTRODUCTION}

There is a growing body of research in the field of HCI on design for indigenous people (see e.g. Coenraad 2019; Lawrence 2017, 2019; Peters 2018: Russel 2015). While the cultural and historical dimensions of this work are acknowledged [1] less is known about the design of digital technologies aimed at reflecting the core of indigenous cultures' interactions with design processes (including design efforts to reflect materials that symbolize and communicate their history, norms, values, rituals and cultural practices). In this paper we present the results of a project aimed at addressing this issue through the design of such digital solutions for indigenous people. At the project level, we explored the design of a web-based digital archive for the Sámi people located in the far north of Scandinavia.

Web-based digital archives are typically built on top of databases to provide access to collections [2]. However, finding media in these archives can be difficult [3]. To address this design challenge, we worked in close collaboration with our target users, and strove to implement functionality that would improve media searching based on searches, filters and shared collections.

Our efforts were focused on designing a web-based digital archive that enabled easy access to collections that reflected the users' cultural and historical heritage. During the development of the digital archive, we noticed that our design process demanded a "double sensitivity". In this paper we present this notion of "double sensitivity", and we describe how we carried out our design research in relation to those sensitivities. We also present a model that illustrates how the two sensitivities are intertwined. At a methodological level, we suggest that this "double sensitivity" comprises 1) having a "cultural sensitivity" in relation to the indigenous people, and 2) having a "design sensitivity" in terms of the design proposals developed.

The paper is structured as follows. First, we present the background and other work related to our project. In this section we focus on existing research on digital archives, digital cultural heritage, and design for indigenous people. We also review the existing literature on cultural and design sensitivity. Second, having outlined the strands of existing related work, we present our project on designing a digital archive for the Sámi people. We present the project, our design efforts, and the user study we conducted. We also report on how we dealt with culturally rooted design challenges, and how we worked with this "double sensitivity" to acknowledge and appreciate the particular context we were designing for. Third, we 
present the results from the user study. Fourth, based on the results, we present our proposed generalized "double sensitivity" model, which illustrates how cultural sensitivity needs to be entwined with design sensitivity to make the final product both functionally and culturally sound for its intended use. Here we illustrate how we have worked back and forth between the conceptual notion of "double sensitivity" and our design case where these two sensitivities were practiced. Finally, we present our conclusions, their implications and suggestions for moving forward.

\section{BACKGROUND \& RELATED WORK}

Our project derived inspiration from prior work involving physical and digital archives, ethical considerations in researching and designing for indigenous groups, and ethical encounters in HCI research and design

\subsection{The Sámi culture}

Following The indigenous population of Sápmi, which includes Norway, Sweden, Finland and parts of Russia are complex to define. A modern definition of who a Sámi person is can be related both to geography (living in a Sámi area) and family (born in a Sámi family or having Sami relatives) but in this day and age there are many definitions of who a Sámi person. A person can be both Sámi and for example Swedish. Earlier laws stated that a person who makes a living of reindeer herding is a Sámi, but this is not true any longer and now anyone with geographical or family bond can identify as Sámi [48].

\subsection{Physical \& Digital Archives}

According to Merriam Webster, Archive, by definition, means "a place in which public records or historical material (such as documents) are preserved or a repository or collection especially of information." Archives are used to investigate various historical contexts, such as medical records, colonial studies and public records [4]. Archives are therefore important institutions that shape our memories [3]. Archives are considered to be a repository of historical sources, and archivists are assumed to be neutral and objective. However, there are a number of researchers that believe that archival documents, institutions and systems do have a tacit narrative that provides meaning to a particular archive [5].

Advances in digital technologies have made drastic changes in the design and development of modern archives. Since the early 2000s more and more archive institutions and newspapers have digitized their resources. Despite this, however, still only a fraction of archival sources has been digitized, and in some countries, such as Sweden and Finland, this is estimated to be only 5 percent. Digital archives are known as platforms that preserve information in a way that can be (re-)discovered, accessed and presented at any time in the future [6]. There are many studies investigating how to develop digital archive platforms for longer and better preservation of the archive materials and their increased use [6] [7]. However, many researchers believe that merely developing a digital platform for preserving archival sources is not enough, and a visual interface for digital archives is necessary to increase the accessibility of such platforms [8]. In a study conducted by Hong et al. it was evident that interface design is an important factor influencing the use of digital archives, and thus the quality of interface design is important in order to promote digital archive usage [9]. Many researchers have evaluated digital and online archives [10] [11], resulting in different guidelines in terms of designing easy access digital archives, such as browsing the archives with minimum mouse clicks, the importance of simple browsing and avoiding downloadable viewers [11]. Digital Archives in relation to indigenous populations can be found in various countries and research suggest that it can be of great importance and complexity to design for [59]. In the field of $\mathrm{HCI}$, various designs of digital archives have been explored, such as the use of autonomous sites in preserving data [2], adopting reflective design [12], interactive systems that foster face to face conversation and story sharing spatial interaction using objects as a way of embodying storytelling [3], and ubiquitous computing and multimedia designs [14]. The design of digital archives and the potential contribution of heritage practice to HCI were explored specifically at a CHI workshop in 2012 [15]. As citizen participation plays a vital role in restoring and preserving cultural heritage, some researchers have explored social platforms that encourage people to act as skilled storytellers by creating personalized stories and sharing them with friends, associates and professionals [16].

\subsection{Ethical Considerations in Researching for Indigenous Groups}

Since the first HCI ethical guidelines were written by Wendy Mackay in 1995, much has changed [31]. In 1995, research involving video was new to the field, and Mackay specifically focused on writing about those challenges. Since the mid-1990s, new tools and techniques for conducting research in novel areas have been developed, which is reflected in the way we generate and understand knowledge in $\mathrm{HCI}$.

As new technologies increasingly pervade various aspects of our life, our attitude towards what methods to use and how to construct knowledge shifts. Thus, ethical considerations are becoming a key issue for HCI researchers in different contexts [32-36]. In particular, as new technologies are designed and evaluated for vulnerable and marginalized participants, $\mathrm{HCI}$ researchers face ethical dilemmas that are highly contextual and very difficult to plan for in advance [33] [58]. Working in these sensitive contexts creates challenges known as 'situational ethics', referring to ethical considerations that emerge while conducting research [37]. However, situational ethics also provide the opportunity to improve ethical review processes by incorporating the new understanding gained from interacting with the context. Therefore, questions such as how HCI research should be conducted and what our common values are as a research community are being addressed by a number of researchers.

Although the majority of these reflections and discussions happen within informal venues, some researchers have published their ethical considerations of different contexts, such as studying animals in HCI [35], and exploring the impacts of town hall-style meetings [32]. Brown etc., have presented five proposals regarding how HCI research should be conducted ethically [36]. In their second proposal, Brown and his team focus on conducting research with vulnerable populations. Their proposal insists that "rather than focus on harm we might instead ask who benefits: Is it just the researchers?". They suggest that interventions with vulnerable 
populations must result in greater benefits for those populations than the researchers [36]. Applying this approach led to our design using double sensitivity for a digital archive that is responsive to the sensitivities of the culture and translates them into design sensitivities. In section 4 we further discuss the full meaning of cultural and design sensitivities. Focusing on these sensitivities from the outset resulted in a platform design that was the most beneficial for the Sámi community.

In going through the existing body of published research we were surprised by the lack of studies focused on design for indigenous people, and in particular the lack of studies aimed at documenting work on the design of digital archives for these user groups. In relation to this existing gap in the literature we see our work as a contribution in how it offers some initial results on this particular topic.

\section{DIGISÀMI: DESIGNING A DIGITAL ARCHIVE FOR THE SÀMI PEOPLE}

The work reported in this paper is a part of a large-scale research program with the primary aim of exploring new technological solutions for the Sámi people. Accordingly, the research program covers web-based solutions and also mobile technologies to enable flexible support across the geographical areas that the Sámi people travel every year. Currently, material regarding the Sámi cultural heritage can be found in several archives and collections, which for historical reasons have been stored in museums and collections across the Nordic countries but also in Europe.

The design process for the digital archive was therefore developed with a diverse user group in mind. The project was, for instance, financed by a program aimed at strengthening the collaboration between Norway, Finland, Sweden and Sámi, and it was therefore crucial that the end product was accessible to all those areas. The results of this project will not only contribute to contemporary and future design research, but will also result in an interactive and published digital archive. Within this digital archive there will be archival material supported by established archive institutions. These institutions aspire to promote their archive material to as wide a target group as possible, which had to be considered in the design.

According to Mikaelsson (2016), the Sámi peoples' lands and territories are linked to their existence and survival. He states, "We (Sámi) are the land and the land is us; we have a distinct spiritual and material relationship with our lands" [21]. Discrimination and exploitation in relation to Sámi people are ongoing in Sweden today, and conducting research about this topic is legitimate and ethical from a Sámi perspective [17]. Because of discrimination and exploitation, there is a need for the Sámi people to demonstrate their existence [38]. Within the Sámi community there are, however, different views on whether or not Sámi history should be made available for a wider audience. Some are proud of it, while others are against marking cultural historical settlements on a map because this is contrary to traditional Sámi culture [38].

How does one design a digital archive with aspects such as these in mind? A digital archive is about storing cultural and historical material and making them easily accessible. This raises both a design challenge and an ethical challenge in this particular context.
Accordingly, the next section covers the methodology applied in this project with an emphasis on these challenges.

\section{METHODOLOGICAL FRAMEWORK}

A good contextual understanding is crucial when designing any system [39], but particularly so when designing for indigenous groups. We wanted to design a digital archive that was not only easy to access and use but included features that were aligned with the specific characteristics of the Sámi cultural heritage and values.

As a group of researchers where none of us was an expert on Sámi culture, we had a massive responsibility. As stated earlier concerning the design of digital archives, it was evident that the majority of the existing digital archives had been designed based on a western epistemology and a western definition of cultural heritage. Designing for sensitive indigenous groups required a different approach. We needed to be sensitive in relation to who we were designing for ("cultural sensitivity") as well as how our design could reflect those needs ("design sensitivity"). Accordingly, we had to work with a "double sensitivity", as outlined below.

"Cultural sensitivity": we had to understand the sensitive setting within which our research was placed, being aware of the cultural sensitivities of the Sámi culture. Understanding the cultural sensitivities played a crucial role in determining which values within the community could be represented in the design in the form of features, functionalities and overall layout.

"Design sensitivity": the design had to be considerate in relation to the cultural values it was intended to support, and in relation to how it could be adapted further to encompass cultural values. This sensitivity included how to address cultural values in the form of appropriate design features and how those features could be adopted by the intended users and the community at large.

In order for us to be aware of these sensitivities, we decided to adopt a "user sensitive inclusive design", approach, as suggested by Newell and Gregor [40]. In order to have a design that is inclusive of sensitivities, the designers need to develop an empathy with the users instead of merely relying on guidelines or lists of requirements. Replacing the word centered with sensitive in "user centered inclusive design" means, according to Newell and Gregor [40], highlighting the fact that it can be hard, if not impossible, to design a product that is accessible and appreciated by all potential users. Being sensitive means being aware of this fact and then trying to achieve something more obtainable, which is inclusive design. This approach has been widely used in designs for elderly people and users with specific challenges [41-43]. In developing the double sensitivity framework, we followed the established tradition in HCI and Participatory Design research to integrate different aspects of sensibilities when working with user groups and design for users. In particular we were inspired by the early work by Friedman [49] on value-sensitive design, and how that perspective has been further developed by Le Dantec et al. [51] and Borning and Muller [50]. We were also inspired by Participatory Design research, in particular Muller and Kuhn [53] and the work by Merritt and Stolterman [55] on cultural hybridity in participatory design, and how that perspective has been applied in similar studies in Namibia by Katjivirue [54]. 
With these two sensitivities as our guiding framework, we strove to establish a close relationship with the Sámi community to provide the context and foundation for the design project. In doing so we sought to increase our knowledge base as much as we could by reading various resources about the Sámi culture, and participating in Sámi events, including the so-called "Sámi week". We visited the Sámi museum frequently to learn more about their history and culture, and we took part in the Sámi film festival in 2019. We even took part in Sami cookery classes to become familiar with the taste of Sámi food and to learn more about their culture. As well as these activities, we held a set of informal interviews with a number of people within the Sámi community from diverse backgrounds. Those informal conversations had a massive impact on our understanding of the cultural sensitivities: they helped us grasp the cultural norms and values of Sami culture, and generated ideas about how we could translate them into our design process, and what that implied for the design of a digital archive that could support our particular context.

\subsection{Empirical Case Study}

Guided by our methodological framework, we carried out a preliminary study, designing and evaluating a web-based digital archive aimed at supporting easy access to materials that reflected Sámi cultural and historical heritage. This prototype shared many features of typical archival web-based digital material. However, because we were aware of the cultural sensitivities, we decided to design features that were mapped to those cultural sensitivities while simultaneously adopting a design-sensitive approach. Below we present this prototype, focusing on three of those features, and then we describe the user study that followed.

4.1.1 Prototype. Our digital archive design shares many features with other online archive websites, including: 1) standard search filters, 2) visual research, 3) login functionality, 4) standard filters to sort the results, and 5) different types of archival material (see figure 1a). In addition to these standard features there are also certain features that were specifically designed to address the "double sensitivity". The three main features are: a) a map, b) a community function that includes the collections, user contributions and stories (although parts of this feature, such as the "stories", were not fully implemented in the prototype, the icon in the top right corner did result in interesting discussions with some of the participants) and c) ethics, which presented a novel way of designing the ethical guidelines of the archive with double sensitivity in mind. In the following section we will discuss these three main features in further detail. Below, we present three of our "designed features that uses double sensitivity", and the design process for each.

The Map: During our informal interviews we asked many different questions about their relationship with their Sámi heritage. We also relied on open-ended questions, e.g. "what are the main characteristics of being a Sámi according to you?", which often resulted in interesting discussions. All of the interviewees mentioned the importance of Sámi land for those belonging to the Sámi culture. In some of the discussions we noticed a desire for a decolonized archive and map. This cultural sensitivity resulted in a feature designed so that geographical borders in the map are options that can be added or removed (see figure 1. b). We provided the border filter as an option for the users in the interests of our broad target group.

The community: We also noticed that many of the Sámi communities were interested in researching their family trees and stories related to their ancestors, and related to the fact that the community is an important entity among the Sámi $[23,44]$ we created the community aspect. This feature included the possibility of creating a personal profile, uploading archival material, creating personal collections and following other popular collections, commenting on material, and liking comments.

This requirement was very evident from the way the Sami younger generation use social media such as Pinterest to share their "Duodji" art. Another example was how Sámi participants in the cookery classes were specifically interested in family recipes. The idea of providing a platform for users to make communities within the overall community is a good example of the features we included in our web-based digital archive using "double sensitivity" in mind: understanding the cultural sensitivity of the community and translating that into a design feature. Such a feature is a great compromise for digital archives designed for indigenous cultures. Indigenous cultures are very effective at preserving their heritage orally, as stories that are told face to face, often with no written trace [45]. Depending on the stories, some are for the general public to hear, while others are only for selective audiences. Therefore, trust is an important aspect in these cultures.

Applying "double sensitivity", we designed the feature so that when you create a profile you can select who you want to share your uploaded material with: just yourself, just those who are following your collections, or with everyone. These features build on the idea that a community can be enriched by private stories told in combination with the material presented in the archive. By being able to add personal material or personal stories it is possible to create an archive that is not only filled with material from governments and other institutions but to have a social archive that is constantly changing and evolving with the help of the individual users.

\subsection{Design Process}

Overall, we spent more than six months conducting background research into understanding the Sámi culture, reflecting on the "cultural sensitivities" and thinking about how they could be translated into "design sensitives". In order to obtain further knowledge about the "double sensitivity" related to our archive, including material presenting Sámi cultural heritage, we initiated our design process by holding a variety of events including multiple brainstorming sessions, workshops and weekly meetings and discussions among the design team. During these six months we organized three larger workshops with representatives for the Sami people, the designers and the Sami archives. These sessions were conducted in parallel with our research into the "cultural sensitivities", so we were able to discuss the design of features that would be in line with those sensitivities. Thus, the opinions of many were taken into consideration, both in relation to the cultural aspects of the archive and the functional design, and the meaning that the features would hold for different groups. Early sketches of the three main features were created in accordance with these "cultural and design sensitivities" 
a)

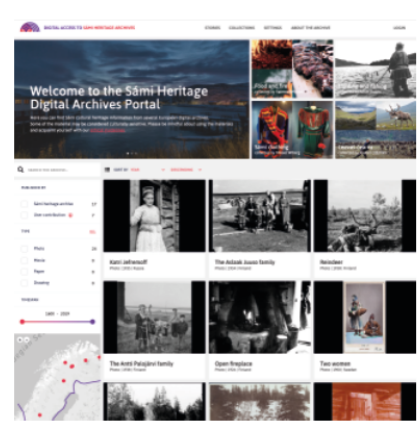

c)

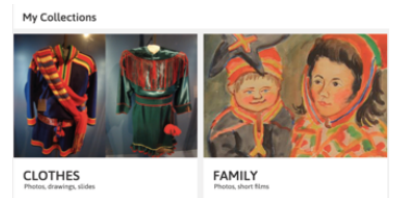

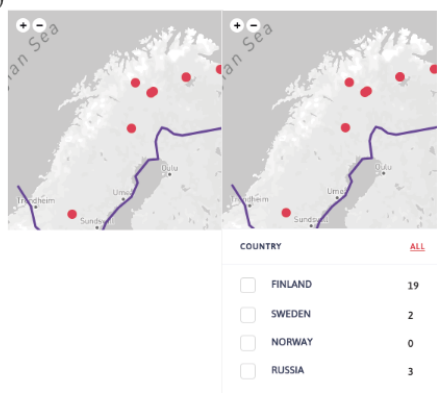

d)

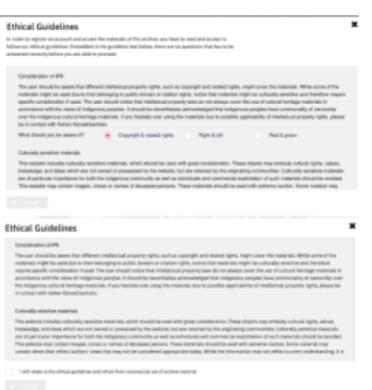

Figure 1: Captions of the prototype and its features. a) The first page of prototype, b) The feature of the map incorporating “double sensitivity" means that border filters are optional, c) The community - a user's collection, d) Ethics: versions of the ethical guidelines with and without questions

that were designed in one of the early brainstorming sessions, are presented in figure 2. Overall, we had to work with iterations that involved interactions with the Sami people, design work, demos, sketches and workshops. We worked back and forth - in order to be sensitive to our users, and in relation to what we designed for this user group.The outcome was our web-based digital design. As seen in figure 1a, it shares many features with other platforms, such as search functions and timeline filters. However, as already mentioned, there are other features designed specifically as a result of the "double sensitivity" approach taken by our project.

\subsection{User study}

We carried out a user study of our web-based digital archive in February and March 2020, comprised of three parts: two interviews and one prototype navigation. The first interview was conducted in order to establish what knowledge the users had about both Sámi culture and archives. Participants then navigated around the prototype. The aim of this part of the user study was to evaluate the general feel of the prototype, specifically the "double sensitivity" features. Initially the participants were asked to navigate the prototype by themselves, in order to get the "look and feel" of it. They were then given different scenarios and tasks to perform. The participants were able to hear instructions from the researcher via the software, and the researchers were able to see the screen and hear the participants. With the participants' consent, their voices and the screen were recorded. In order to ascertain the participants' thoughts on the process, they were asked to conduct the navigation using the think-aloud method [46].

After the evaluation, the participants were invited back for a second interview to explore their experience of the prototype. This interview consisted of questions focusing mainly on their overall opinions relating to their personal experiences with either Sámi culture or archives, as well as more detailed aspects and features of the prototype. Anything that came up during the first interview or trial navigation was followed up during this interview.

4.3.1 Participants. In order to establish a good basis for the study, some parameters were established to incorporate both a rich dataset of material and a broad target group for the web-based digital archive. In total we interviewed 12 participants, three from each specific category (see table 1). These categories were created before the user study to make sure that the participants encompassed different experiences across different fields, thus enabling the essential aim of the user tests. The main categories were knowledge about Sámi culture and knowledge about archives. Knowledge about Sámi culture would include individuals who were themselves a part of the Sámi community and or had great knowledge of the community, this could for example be phd-students writing about a certain part of the Sámi culture. Individuals that was part of the knowledge about archives group where individuals who had extensive knowledge about archives, both physical and digital. Examples of individuals within this group was archivists, students and phdstudents who all had used archives for research purposes during 


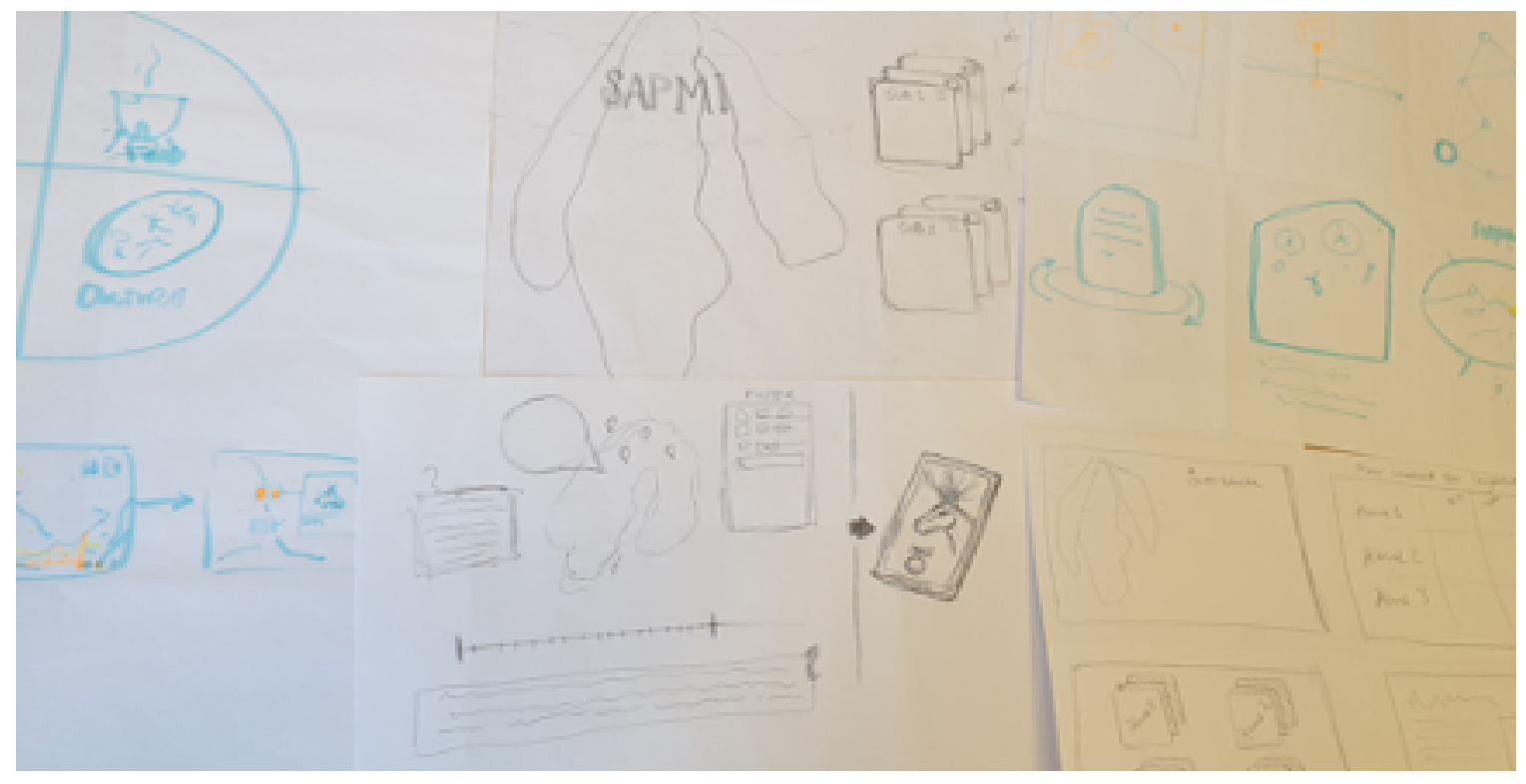

Figure 2: An example of some sketches made during the brainstorming session

Table 1: Distribution of participants in the user study

\begin{tabular}{lll}
\hline & Knowledge about the Sámi culture & Limited knowledge about the Sámi culture \\
\hline Experience and knowledge of archives & Group 1. & Group 2. \\
& 3 participants & 3 participants \\
& $(3$ Male $)$ \\
& $(2$ originally $)$ & \\
Limited experience and knowledge with & Group 3. & Group 4. \\
archives & 3 Participants & 3 participants \\
& $(2$ Male \& 1 Female) & $(2$ Male \& 1 female) \\
& $(2$ originally from Sámi community) & \\
\hline
\end{tabular}

many years. Individuals that was part of the group of no-sami nor no-archive knowledge was not a part of the Samí community, nor had any further knowledge of it, and they did not have much experience with any type of archives. All of the individuals within this last group was students from different fields. A table over the user groups are given in table 1

\section{RESULTS}

The data recorded from the interviews and the participants' interaction with the prototype were analyzed using thematic analysis as the methodological approach [47]. Five main code clusters were identified: tonality of the design, usability, sociability, ethical considerations and finally technical errors. The code technical errors merely focused on technical issues and glitches with the prototype, and we will not expand on this code further here. In the following sections we present the results from the four other codes.

\subsection{Tonality of design}

Some questions were raised and opinions expressed regarding the overall design and look and feel of the prototype. They included color use, placement of certain elements, phrasing of certain words and other design-related aspects. The code tonality of design was created to collate all the opinions regarding design. Some participants were positive about the general look and feel of the prototype. Participant 2 said "It generally felt nice, I don't know in what way but it felt nicer and more sympathetic". Participant 7 was also positive and said "The prototype feels more designed than a conventional archive". Some participants felt that the accessibility of the prototype was good and that they could also understand what the limitations were. Participant 9 said "I grasped better what could be done in this (prototype) just by looking at it". Participant 8 also mentioned this, "I understand what the prototype can give me".

\subsection{Usability concerns}

The code usability refers to any comment, question or issue raised by the participants in regard to the usability aspects of the prototype. This code was then divided into two subcodes:

The first one called usability Context encompassed comments that were more focused on the context of the archive. These were 
Table 2: Table over participants and their level of knowledge in regards to archives and Sámi culture

\begin{tabular}{|c|c|c|c|}
\hline Participant no. & User group & Knowledge about Sámi culture & Knowledge about archives \\
\hline 1. & Group 2. & Limited knowledge & $\begin{array}{l}\text { Has extensive knowledge of } \\
\text { both physical and digital } \\
\text { archives through studies. }\end{array}$ \\
\hline 2. & Group 3. & Identifies as a Sámi & Limited knowledge \\
\hline 3. & Group 4. & Limited knowledge & Limited knowledge \\
\hline 4. & Group 4. & Limited knowledge & Limited knowledge \\
\hline 5. & Group 1. & $\begin{array}{l}\text { Identifies as a Sámi and studies } \\
\text { Sámi culture }\end{array}$ & $\begin{array}{l}\text { Has extensive knowledge of } \\
\text { both physical and digital } \\
\text { archives through studies. }\end{array}$ \\
\hline 6. & Group 1 & $\begin{array}{l}\text { Identifies as a Sámi and works } \\
\text { with Sámi culture }\end{array}$ & $\begin{array}{l}\text { Has extensive knowledge of } \\
\text { both physical and digital } \\
\text { archives through studies. }\end{array}$ \\
\hline 7. & Group 2. & Limited knowledge & $\begin{array}{l}\text { Has extensive knowledge of } \\
\text { both physical and digital } \\
\text { archives through studies. }\end{array}$ \\
\hline 8. & Group 1. & Studies Sámi culture & $\begin{array}{l}\text { Has extensive knowledge of } \\
\text { both physical and digital } \\
\text { archives through studies. }\end{array}$ \\
\hline 9. & Group 4. & Limited knowledge & Limited knowledge \\
\hline 10. & Group 3. & Identifies as a Sámi & Limited knowledge \\
\hline 11. & Group 2. & Limited knowledge & $\begin{array}{l}\text { Has extensive knowledge of } \\
\text { both physical and digital } \\
\text { archives through studies. }\end{array}$ \\
\hline 12 & Group 3. & $\begin{array}{l}\text { Grew up in a predominantly } \\
\text { Sámi area and claims to have } \\
\text { large second hand knowledge }\end{array}$ & Limited knowledge \\
\hline
\end{tabular}

more common among those participants who had more experience of archives.

The second one called Usability Functionality encompassed comments that covered general aspects of usability, such as adding a specific button.

In going through the usability concerns we were inspired by the work by Díaz et al [56] on cultural-oriented usability heuristics in order to link aspects of usability to the particular cultural context in focus.

Many of the researchers and participants with archival knowledge would comment that context was a very important feature to them. The Usability Context code was applicable to various responses made by different participants. For example, participant 11 explicitly mentioned "I want to have the volumes in front of me when I open the boxes (in physical archives) and when I go to a digital archive I want the same thing”. Many similar comments were made by a number of the participants in relation to any digital archive, not just our web-based digital archive.

Given that we were aiming for a minimalistic design for our prototype, we decided to avoid some archival technical features, for example identifying the organization from which the material came. Although this minimalistic design was appreciated by the non-professional participants, those participants who had extensive experience of archives felt that this feature resulted in a lack of context.
Additionally, a large number of participants mentioned struggling to get a feeling of context when using a digital archive. All of the participants with knowledge of archives said that the best aspect of physical archives was the context provided by seeing, smelling and feeling the material. However, the more positive aspects of having a digital archive are availability and searchability. As participant 5 said about physical archives, "you feel the material, you have the sense of feel and touch of the material, and you have the smell. When you work on a computer you only have the visual, and also when it comes to the visual, I would say that it is one thing to see the document on a web page and another thing to see it in real life."

The part of the archive that included a map where participants could navigate to various geographical locations also generated comments that were coded as usability. The map was presented with two different designs, one contained country or border filters, where users could see the Sámi countries, while the other design did not contain the same filters and users had to navigate dots on the map in order to find a certain geographical location. Opinions on this were many. Some participants said that for them the map was more usable with the country filters, because they did not have any detailed knowledge of the borders and did not see any issues with having the borders there. This was expressed, for example, by participant 12: "The map would be confusing for a person with a non-Scandinavian background". However, while some of the participants, such as 
participant 1, appreciated the idea of the borderless map as a symbol of a borderless world, others highlighted the importance of raising this as an issue. For example, participant 11 mentioned that "The border issue can be a discussion he sees the point of having it this way although it is not an issue for me." Most participants did not see why there would not be any borders or country filters, simply because for them it was easier to have them than to not have them. Participant 11 suggested that having the borders or countries merely depended on the user: "If a user knows about the borders, then they don't have to be there but if a user doesn't then the borders are better, it all depends on the user".

The part of the archive that included a map where participants could navigate to various geographical locations also generated comments that were coded as usability. The map was presented with two different designs, one contained country or border filters, where users could see the Sámi countries, while the other design did not contain the same filters and users had to navigate dots on the map in order to find a certain geographical location. Opinions on this were many. Some participants said that for them the map was more usable with the country filters, because they did not have any detailed knowledge of the borders and did not see any issues with having the borders there. This was expressed, for example, by participant 12 : "The map would be confusing for a person with a non-Scandinavian background". However, while some of the participants, such as participant 1, appreciated the idea of the borderless map as a symbol of a borderless world, others highlighted the importance of raising this as an issue. For example, participant 11 mentioned that "The border issue can be a discussion he sees the point of having it this way although it is not an issue for me." Most participants did not see why there would not be any borders or country filters, simply because for them it was easier to have them than to not have them. Participant 11 suggested that having the borders or countries merely depended on the user: "If a user knows about the borders, then they don't have to be there but if a user doesn't then the borders are better, it all depends on the user".

\subsection{Sociability features}

This code referred to comments, issues and questions raised by the participants in regard to the social aspects of the prototype, in particular the design of the sociability feature. Given the unconventional nature of this feature, opinions about the social or community side of the prototype were both positive and negative. Most participants discussed how hard they thought it would be to have a comments function in the archive. Allowing comments would mean having strong moderation to mitigate discriminating comments, and some participants discussed whether that would be possible or even worth it. Participant 12 felt that moderation would simply be too much: "I just don't see it being worth it, it would be hard to moderate all the hate". The majority of participants felt that even if there was extensive moderation and restrictions, the value of being able to share experiences and thoughts on the archive was greater than the possibly negative consequences.

In spite of this, the majority of the participants responded positively to being able to save images to private collections and expressed how useful they thought such a feature would be. Participant 8 liked the save feature, "I very much like the fact that I can save the images like on Pinterest" and also felt like it would be useful to be able to comment and share the feature, "I would use the social aspect of the site, it would give value to my work". Participant 5 felt the feature could bring a sense of community: "it was more like a community type of webpage, like this is for people interested in like Sámi photography”.

Only a few of the participants did not find this feature useful. Participant 2 preferred to use his own methods to save the material: "I can see why some might use it but for me I would rather use my own personal methods of saving and collecting the material". Participant 4 also had a hard time seeing why this function would be good: "What is the point? I cannot see the point for myself, but perhaps for elders it would be good".

Overall the social aspect was positively received by the participants, even if some questions were raised, particularly about the commenting function because this would require significant moderation by the administrators of the archive.

\subsection{Ethical considerations}

Authors The code ethical considerations encompassed comments that were mostly directed towards the ethical guidelines and how the participants experienced this feature.

Reactions to the two different ways of presenting the ethical guidelines varied among the participants. The ethical guidelines of both the "normal" wall of text and the "quiz" version were appreciated by some and not liked so much by others. Participant 6 talked about the version with questions: "Most people won't read the guidelines, and this will make me not want to join the archive". Participant 2 also felt the questions were not a good idea: "The questions feel too easy and dumb, like the answers are given without any thought". However, there were positive comments as well. Although some participants raised concerns, others liked the idea of having a new ethical guidelines solution. Participant 1 said, "It's a good way for users to have to read the ethical guidelines, the questions are also good. Without questions I will not read guidelines". Participant 3 also liked the design: "I like the design of the questions".

Participant 7 questioned how the text of the ethical guidelines was written and said that it could possibly strengthen prejudice against the Sámi population. "The text is interesting; you need to talk to different Sami-groups and not just the groups that the government defines as Sámi. The sentences can be seen as portraying us like victims". This was because the phrasing of the text looked like it was written by a non-Sámi individual who wanted to create a helpful text. "This text looks like it was written by a non-Sámi person who wants to do things right." Further, and regarding how images in the archive were named, participant 5 mentioned the ethics of naming places and individuals: "There are a lot of pictures of anonymous Sámi people, and it's a good way (having comments) to name places, so it's not just oh, random Sámi in a random mountain area, it's a way to empower and to say, okay this is the place, it has a name and a person".

This section has presented the highlights of the results generated by our user study. These results provided a means to uncover opinions about the features of the archive. The opinions expressed could then be summarized and translated into a model in order to show 


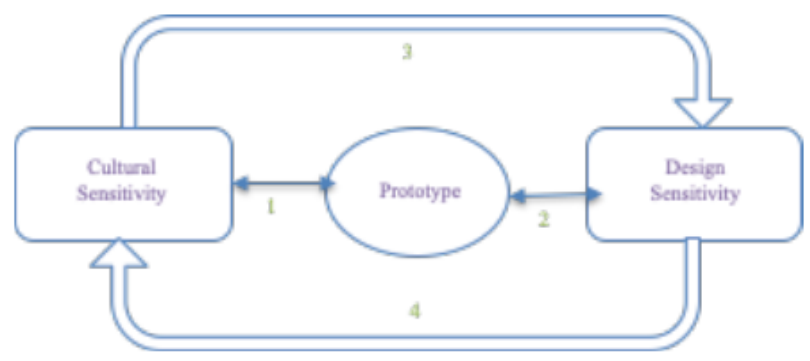

Figure 3: The "double sensitivity" model

how "double sensitivity" has benefited the design of this prototype and how this study has helped us understand "cultural sensitivity" better. In other words, the model illustrates how using "double sensitivity" can be beneficial in the design of prototypes such as this archive. In the next section we present the "double sensitivity" model followed by discussion on the overarching take-aways for HCI researchers and designers. technologies.

\section{THE “DOUBLE SENSITIVITY” MODEL}

Based on what we have described, the core of this research and study is to take account of sensitivity. This sensitivity is defined in two ways: "Cultural Sensitivity" and "Design Sensitivity". Based on our empirical findings, and our design project conducted in close collaboration with the Sami people we propose that these two notions are fundamentally entangled. Accordingly, the model in fig 2 shows how taking a sensitive approach to a user group can result in the design of an archive that, in our case, can be appropriate and appreciated by the community. As illustrated in figure 3, the "double sensitivity" model consists of four parts.

1. "Cultural sensitivity" feeds back to the design of the prototype, so that, based on the knowledge gained by understanding the sensitivities within the culture, we can design features that are suitable for that culture. However, this is itself an iterative process that occurs during the design process.

2. "Design sensitivity" provides feedback to the prototype. During the design process several choices have to be made to implement the desired features. Design sensitivity is also an iterative process that modifies the prototype based on the context.

3. The more knowledge we have about the embedded cultural sensitivities, and the more time we spend learning about the context, the better we understand the "design sensitivities".

4. Similarly, the more we know about the sensitives within the design, the easier it is to identify the sensitivities of the culture. In other words, the prototype functions as a means to understand the "cultural sensitivities" better.

Stages 3 and 4 are considered to be feedback loops. Discussing our prototype with the Sámi community, and their realization of our cultural and design sensitivities, resulted in established trust between both sides. This trust resulted it engagement that in return had an impact on our design. Therefore, the design of the archive and the user study both helped us shape our sensitivity in designing for the Sámi community.

\section{DISCUSSION}

Authors This study has generated many interesting takeaways, both about sensitivity and with this the double sensitivity model which relating to our research. Being sensitive in an interaction design project is always challenging. We are supposed to understand things, but also have to change or introduce new things within the context we are trying to be sensitive about. There are many things that need to be taken into consideration in order to be sensitive - from understanding user needs, capabilities and values, to the project requirements, to aspects of how the system might support those needs, and how the design might redefine practice once it is implemented. From that perspective Interaction Design (IxD) is always about this need to be sensitive to the context, and about how the design works in relation to that particular context. However, while it is easy to state that one should always be sensitive about user needs, and the particular context in question, little has been published about how these sensitivities play out during the design process.

We explicitly departed from well-known aspects of HCI design research to make visible the sensitivities needed to design interactive systems successfully in relation to use contexts where such sensitivities are key issues. In our work we have made two such sensitivities visible, i.e. we applied the "double sensitivity" approach proposed in this paper. We will now reflect on these two sensitivities and its implications in relation towards our study.

"Cultural sensitivity" - By focusing on a context with clear needs, and important values to protect, we have in this project been able to make the importance of a "cultural sensitivity" visible. We worked with "cultural sensitivity" throughout the project - from the initial studies, to the ethics workshops, to the design work. As already mentioned, "cultural sensitivity" is about understanding the sensitive setting within which the research is situated, which for us was being aware of the cultural sensitivities within the Sámi culture that formed the basis of our research. In some examples individuals who identified as Sámi did not think that a number of features were a good idea, but non-Sámi individuals did, for example, the ethical guideline questions. This is an interesting result 
and it points us to a number of possible conclusions. Either the questions of the feature are not fully developed, or the concept it-self needs work done. There is also the possibility that this "culturally sensitive" feature is un-necessarily sensitive as one Sámi-participant expressed that in reality people don't like to read long texts and as a result the service will not be used despite the text being in good will. However, there are clear statements from representatives of the Sámi community that being generally sensitive around related aspects of the Sami community is of utter importance. In this case it is possible that the effort of designing sensitively did not translate to the individual or that the feature demanded more iterations to translate better. Changing the current form of the feature may lead to other outcomes but this result also taps into a slightly larger question that requires further research: Is it possible to design sensitively to the point of exaggeration?

Implications: Having knowledge about "cultural sensitivities" can play a crucial role in preventing insensitive design features. It can also be of value within the community as it builds up trust in relation to a design project that might expose cultural values.

"Design sensitivity" is the second sensitivity we made visible in this project. This is about making design choices sensitively and being reflective about how the design relates to the context it is intended to support. We have demonstrated this in how we approached the implementation of the ethical guidelines, which was widely discussed by the participants showing large potential for its development. The general concept of the guidelines presented in this study was appreciated although it became clear that some of the details have to evolve in future research. We also made the collections visible through our web-based digital archive.

Implications: "Design sensitivity" comes with a responsibility for the designer. It is about both acknowledging the context, values and culture that the design is intended to support, and also making sure that this is reflected in the design. The proof lies in the making and, accordingly, someone's "design sensitivity" needs to be evaluated by examining the design from that perspective.

As we have illustrated in our model (figure 3), these two sensitivities are fundamentally intertwined. Our sensitive understanding of context ("cultural sensitivity") informs our design work, and our design reflects to what extent we have such "cultural sensitivity". If the design corresponds with the "cultural sensitivity", we can say that our design demonstrates a "design sensitivity" in how it acknowledges the context it is designed to support. While these two sensitivities are well-known aspects in $\mathrm{HCI}$ and participatory design research we suggest that the notion of "double sensitivity" might contribute to these two strands by providing a concept that underscores the importance of unifying these two strands in the process of HCI design research.[54]

As the results from the user study illustrate, we worked simultaneously with both of these two sensitivities as we moved back and forth between a sensibility for the user group, and a sensibility for how we designed for these users. In doing so we managed to acknowledge the culture, values and people we are designing for ("cultural sensitivity"), and our user study indicated that we also managed to reflect that in our design (a matter of "design sensitivity"). Accordingly, we see this model and the identified implications as important take-aways for designers who seek to explore interaction design for sensitive user groups. Further, we believe that this model works in the context of designing digital solutions for sensitive contexts, and we suggest that a topic for future work could be applying this model to other projects and examining its wider applicability.

\section{CONCLUSIONS}

We have presented our work on the design and evaluation of a web-based digital archive. The aim of this research project was to explore ways of enabling easy access to materials about their cultural heritage for indigenous people. On a more specific level, we have reported on our ongoing project to provide the Sámi community with such a digital archive. On a more general level, we have focused on two sensitivities that we became aware of during our project. Through the design and evaluation of a prototype for the digital archive, we noticed how these two sensitivities were intertwined, and accordingly we refer to them as a "double sensitivity". In addition, we have unpacked the notion of double sensitivity by 1 ) reviewing previous work on cultural and design sensitivity that situates our work within the established body of research, 2) illustrating how these two sensitivities are addressed in our project, and 3) presenting a "double sensitivity" model that conceptualizes the relationship between these two sensibilities. We suggest that this model could be useful as a framework for further design research, in particular when designing for indigenous people where there is a need to situate a "design sensitivity" in relation to cultural values. Our design approach is well-aligned with an interaction design agenda that builds upon participation, cultural sensitivity, and ethical considerations, and we suggest that this is a sensible way of moving forward.

\section{ACKNOWLEDGMENTS}

We thank the reviewers for their important feedback. We also thank all of the members of the "Digital Access to Sámi Heritage Archives" research project. Finally, we would like to gratefully acknowledge the grants from Interreg Nord - European Regional Development Fund and Region Västerbotten who made this project possible in the first place.

\section{REFERENCES}

[1] Chris Lawrence, Tuck Wah Leong, Margot Brereton, Jennyfer Lawrence Taylor, Nic Bidwell, and Greg Wadley. 2019. Indigenous HCl: Workshop at OzCHI 2019, Perth. In $<i>$ Proceedings of the 31st Australian Conference on Human-ComputerInteraction $</ \mathrm{i}>\left(<\mathrm{i}>\mathrm{OZCHI}{ }^{\prime} 19</ \mathrm{i}>\right)$. Association for Computing Machinery, New York, NY, USA, 17-19. DOI:https://doi.org/10.1145/3369457.3369553

[2] Cooper, B. and H. Garcia. Creating trading networks of digital archives. in Proceedings of the 1st ACM/IEEE-CS joint conference on Digital libraries. 2001.

[3] Blezinger, D. and E. Van Den Hoven. Storytelling with objects to explore digital archives. in Proceedings of the European Conference on Cognitive Ergonomics. 2016.

[4] Stoler, A.L., Colonial archives and the arts of governance. Archival science, 2002. 2(1-2): p. 87-109.

[5] Ketelaar, E., Tacit narratives: the meanings of archives. Archival science, 2001. 1(2): p. 131-141.

[6] Ludäscher, B., R. Marciano, and R. Moore, Preservation of digital data with selfvalidating, self-instantiating knowledge-based archives. ACM Sigmod Record, 2001. 30(3): p. 54-63.

[7] Song, S. and J. JaJa. ACE: a novel software platform to ensure the long term integrity of digital archives. in Archiving Conference. 2007. Society for Imaging Science and Technology.

[8] Ruecker, S., M. Radzikowska, and S. Sinclair, Visual interface design for digital cultural heritage: A guide to rich-prospect browsing. 2011: Ashgate Publishing, Ltd. 
[9] Hong, J.-C., et al., Applying the technology acceptance model in a study of the factors affecting usage of the Taiwan digital archives system. Computers \& Education, 2011. 57(3): p. 2086-2094.

[10] Gilliland-Swetland, A.J., Evaluation design for large-scale, collaborative online archives: Interim report of the Online Archive of California Evaluation Project. Archives and Museum Informatics, 1998. 12(3-4): p. 177-203.

[11] Maxwell, A., Digital archives and history research: feedback from an end-user Library Review, 2010.

[12] Stanton, R., L. Vaughan, and J. Yuille. Exploring reflective design: an approach to digital archives. in Design Experiments, The Nordic Design Research Conference. 2013. The Royal Danish Academy of Fine Arts.

[13] Shen, C., N. Lesh, and F. Vernier, Personal digital historian: story sharing around the table. interactions, 2003. 10(2): p. 15-22.

[14] Stevens, M.M., et al., Getting into the Living Memory Box: Family archives \& holistic design. Personal and Ubiquitous Computing, 2003. 7(3-4): p. 210-216.

[15] Giaccardi, E., E. Churchill, and S. Liu, Heritage matters: designing for current and future values through digital and social technologies, in CHI'12 Extended Abstracts on Human Factors in Computing Systems. 2012. p. 2783-2786.

[16] Lim, V., et al., PLUGGY: A pluggable social platform for cultural heritage awareness and participation, in Advances in Digital Cultural Heritage. 2018, Springer p. $117-129$.

[17] Drugge, A.-L., Ethics in indigenous research: past experiences-future challenges 2016: Vaartoe-Centre for Sami Research.

[18] Hudson, M., et al., The development of guidelines for indigenous research ethics in Aotearoa/New Zealand. 2016.

[19] Hudson, M.L. and K. Russell, The Treaty of Waitangi and research ethics in Aotearoa. Journal of Bioethical Inquiry, 2009. 6(1): p. 61-68.

[20] Cram, F., Preliminary discussions with Māori key informants. Review of the current processes for ethical review of health and disability research in New Zealand: Report to the Minister of Health, 2003: p. 10.

[21] Mikaelsson, S., We, the Saami, are one People, united in our own culture, lan guage and history, living in areas which since time immemorial and up to historical times, we alone inhabited and utilized. Ethics in Indigenous Research: Past Experiences-Future Challenges, 2016.

[22] Huntington, H.P., S. Gearheard, and L.K. Holm, The Power of Multiple Perspectives: Behind the Scenes of the Siku-Inuit-Hila Project, in SIKU: Knowing Our Ice. 2010, Springer. p. 257-274.

[23] Tervo, H. and M. Nikkonen, «In the Mountains One Feels like a Dog off the Leash»-Sámi Perceptions of Welfare and its Influencing Factors. Vård i Norden, 2010. 30(4): p. 9-14.

[24] Huntington, H., et al., Matching traditional and scientific observations to detect environmental change: a discussion on Arctic terrestrial ecosystems. Ambio, 2004: p. 18-23.

[25] Allemann, L. and S. Dudeck, Sharing oral history with Arctic indigenous communities: Ethical implications of bringing back research results. Qualitative Inquiry, 2019. 25(9-10): p. 890-906.

[26] Drugge, A.-L., How can we do it right? Ethical uncertainty in Swedish Sami research. Journal of Academic Ethics, 2016. 14(4): p. 263-279.

[27] Lawrence, C., et al. \# thismymob: digital land rights and reconnecting indigenous communities. in Proceedings of the 29th Australian Conference on ComputerHuman Interaction. 2017.

[28] Peters, D., et al. " Participation is not enough" towards indigenous-led co-design. in Proceedings of the 30th Australian Conference on Computer-Human Interaction. 2018.

[29] Gunn, M., H. Bai, and P. Sasikumar, Come to the Table! Haere mai ki te tēpu!, in SIGGRAPH Asia 2019 XR. 2019. p. 4-5.

[30] Johnsson, L., et al., Making researchers moral: Why trustworthiness requires more than ethics guidelines and review. Research Ethics, 2014. 10(1): p. 29-46.

[31] Mackay, W.E. Ethics, lies and videotape. . .. in Proceedings of the SIGCHI Conference on Human Factors in Computing Systems. 1995.

[32] Frauenberger, C., et al. Research Ethics in HCI: A town hall meeting. in Proceedings of the $2017 \mathrm{CHI}$ Conference Extended Abstracts on Human Factors in Computing Systems. 2017.

[33] Davis, H. and J. Waycott. Ethical encounters: HCI research in sensitive and complex settings. in Proceedings of the Annual Meeting of the Australian Special Interest Group for Computer Human Interaction. 2015.

[34] Waycott, J., et al. Ethical encounters in HCI: Research in sensitive settings. in Proceedings of the 33rd Annual ACM Conference Extended Abstracts on Human
Factors in Computing Systems. 2015

[35] Väätäjä, H.K. and E.K. Pesonen, Ethical issues and guidelines when conducting HCI studies with animals, in CHI'13 Extended Abstracts on Human Factors in Computing Systems. 2013. p. 2159-2168.

[36] Brown, B., et al. Five provocations for ethical HCI research. in Proceedings of the 2016 CHI Conference on Human Factors in Computing Systems. 2016.

[37] [37]Munteanu, C., et al. Situational ethics: Re-thinking approaches to formal ethics requirements for human-computer interaction. in Proceedings of the 33rd Annual ACM Conference on Human Factors in Computing Systems. 2015.

[38] Roche, G., H. Maruyama, and A. Virdi Kroik, Indigenous Efflorescence. 2018: ANU Press.

[39] Beyer, H. and K. Holtzblatt, Contextual design. interactions, 1999. 6(1): p. 32-42.

[40] Newell, A.F. and P. Gregor. "User sensitive inclusive design"-in search of a new paradigm. in Proceedings on the 2000 conference on Universal Usability. 2000.

[41] Frank Lopresti, E., A. Mihailidis, and N. Kirsch, Assistive technology for cognitive rehabilitation: State of the art. Neuropsychological rehabilitation, 2004. 14(1-2): p. 5-39.

[42] Gregor, P., A.F. Newell, and M. Zajicek. Designing for dynamic diversity: interfaces for older people. in Proceedings of the fifth international ACM conference on Assistive technologies. 2002.

[43] Eisma, R., et al., Early user involvement in the development of information technology-related products for older people. Universal Access in the Information Society, 2004. 3(2): p. 131-140.

[44] Bals, M., et al., Self-reported internalization symptoms and family factors in indigenous Sami and non-Sami adolescents in North Norway. Journal of adolescence, 2011. 34(4): p. 759-766.

[45] Driscoll, C. and M. McKee, Restorying a culture of ethical and spiritual values: A role for leader storytelling. Journal of Business Ethics, 2007. 73(2): p. 205-217.

[46] Van Someren, M., Y. Barnard, and J. Sandberg, The think aloud method: a practical approach to modelling cognitive. 1994: Citeseer

[47] Guest, G., K.M. MacQueen, and E.E. Namey, Applied thematic analysis. 2011: Sage Publications.

[48] Samer.se (2020). Vem är Same? Gathered 2020-08-04 from: http://www.samer.se/ 1147

[49] Friedman, B., Hendry, D., \& Borning, A. (2017). A Survey of Value Sensitive Design Methods. Foundations and Trends in Human-Computer Interaction, 11(2), 63-125.

[50] Borning, A \& Muller, M. 2012. Next steps for value sensitive design. In Proceedings of the SIGCHI Conference on Human Factors in Computing Systems (CHI '12). Association for Computing Machinery, New York, NY, USA, 1125-1134.

[51] Christopher A. Le Dantec, Erika Shehan Poole, and Susan P. Wyche. 2009. Values as lived experience: evolving value sensitive design in support of value discovery. In Proceedings of the SIGCHI Conference on Human Factors in Computing Systems (CHI '09). Association for Computing Machinery, New York, NY, USA, 1141-1150.

[52] Iversen, O, Halskov K \& Wah Leong, T. 2010. Rekindling values in participatory design. In Proceedings of the 11th Biennial Participatory Design Conference (PDC '10). Association for Computing Machinery, New York, NY, USA, 91-100.

[53] Muller, J.M \& Kuhn, S. 1993. Participatory design. Commun. ACM 36, 6 (June 1993), 24-28.

[54] Katjivirue, M. 2014. Participatory design in Namibia. In Proceedings of the 13th Participatory Design Conference: Short Papers, Industry Cases, Workshop Descriptions, Doctoral Consortium papers, and Keynote abstracts - Volume 2 (PDC '14). Association for Computing Machinery, New York, NY, USA, 127-128.

[55] Merritt, S \& Stolterman, E. 2012. Cultural hybridity in participatory design. Proceedings of the 12th Participatory Design Conference: Exploratory Papers, Workshop Descriptions, Industry Cases - Volume 2 (PDC '12). Association for Computing Machinery, New York, NY, USA, 73-76.

[56] Díaz, J, Rusu, C, Pow-Sang, A \& Roncagliolo, S. 2013. A cultural-oriented usability heuristics proposal. In Proceedings of the 2013 Chilean Conference on Human - Computer Interaction (ChileCHI '13). Association for Computing Machinery, New York, NY, USA, 82-87.

[57] Russell, L. 2005. Indigenous knowledge and archives: accessing hidden history and understandings. Australian academic \& research libraries, 36(2), 161-171.

[58] Maunder, A., Marsden, G., Gruijters, D., \& Blake, E. 2007. Designing interactive systems for the developing world - reflections on user-centred design. 1-8.

[59] Christen, K. (2008). Working Together: Archival Challenges and Digital Solutions in Aboriginal Australia. 8(2), 21-24. 